TRANSACTIONS OF THE

AMERICAN MATHEMATICAL SOCIETY

Volume 358, Number 2, Pages 727-741

S 0002-9947(05)03662-7

Article electronically published on September 22, 2005

\title{
CUSP SIZE BOUNDS FROM SINGULAR SURFACES IN HYPERBOLIC 3-MANIFOLDS
}

\author{
C. ADAMS, A. COLESTOCK, J. FOWLER, W. GILLAM, AND E. KATERMAN
}

\begin{abstract}
Singular maps of surfaces into a hyperbolic 3-manifold are utilized to find upper bounds on meridian length, $\ell$-curve length and maximal cusp volume for the manifold. This allows a proof of the fact that there exist hyperbolic knots with arbitrarily small cusp density and that every closed orientable 3-manifold contains a knot whose complement is hyperbolic with maximal cusp volume less than or equal to 9. We also find particular upper bounds on meridian length, $\ell$-curve length and maximal cusp volume for hyperbolic knots in $\mathbb{S}^{3}$ depending on crossing number. Particular improved bounds are obtained for alternating knots.
\end{abstract}

\section{INTRODUCTION}

Given a knot $K$ in a compact 3 -manifold $\mathrm{M}$ such that $M-K$ is hyperbolic, there is a well-defined maximal cusp $C$ in the 3-manifold. The interior of $C$ is a neighborhood of the missing knot such that the pre-image of $C$ in hyperbolic 3space $\mathbb{H}^{3}$ is a union of horoballs with disjoint interiors and such that some horoballs in this set are tangent to one another. Hence the cusp cannot be expanded without causing the horoballs in the cover to overlap in their interiors. There is a welldefined meridian length $|m|$ in the cusp boundary, corresponding to the length of the shortest representative of a meridian in that boundary. A nontrivial curve that crosses $m$ once is called an $\ell$-curve, and the shortest representative of an $\ell$-curve in the maximal cusp boundary has length denoted $|\ell|$. The volume in the maximal cusp of a hyperbolic 3-manifold $M$ is denoted $V_{c}(M)$ and by straightforward calculations, one can verify that $V_{c}(M)$ is exactly one-half the area of $\partial C$. That area is bounded above by $|m||\ell|$. Note that any nontrivial curve in the maximal cusp boundary has length at least one and the only curve that has length exactly one is the meridian of the figure-eight knot complement (cf. Ada02]).

The Gromov-Thurston $2 \pi$ Theorem implies that for a knot in the 3-sphere, $|m| \leq$ $2 \pi$. This was improved by Agol Ago00 and Lackenby Lac00 to $|m| \leq 6$. (They proved substantially more, but this is one implication of their work.) For knots of 10 or fewer crossings, the largest value of $|m|$ is 2.051201, which occurs for the $10_{153}$ knot, calculations of which come from SNAPPEA (cf. Wee]), Jeffrey Weeks's hyperbolic structures program. The largest values of $|m|$ currently known appear

Received by the editors October 2, 2002 and, in revised form, March 1, 2004.

2000 Mathematics Subject Classification. Primary 57M50.

This research was supported by the National Science Foundation under grant numbers DMS9820570 and DMS-9803362. 
in Ago00 and come from a sequence of knots with meridian length approaching 4 from below.

In this paper, we utilize continuous maps of surfaces into knot complements to obtain a variety of bounds on meridian length, $\ell$-curve length and maximal cusp volume for knots. In addition, we prove that any compact orientable 3-manifold contains a hyperbolic knot such that the complement has maximal cusp volume at most 9 . In order to obtain these results, we look at proper continuous maps of punctured surfaces into knot complements. The ideas presented here build on work of Zheng-Xu He [He98], where the author used continuous maps of punctured disks into knot complements to obtain lower bounds on the crossing number of satellites of hyperbolic knots in terms of the meridian length and area of the maximal cusp boundary of the hyperbolic knot. In Ago00, Ian Agol generalized He's constructions and found upper bounds on slope lengths in cusp boundaries in terms of the Euler characteristic of an essential surface punctured by the cusp. Agol also found an upper bound on the number of slopes on the cusp that do not yield hyperbolike (irreducible with infinite word-hyperbolic fundamental group) 3-manifolds after Dehn filling. One implication of Agol's work is the upper bound of 6 on meridian length mentioned above.

We look at the general case of proper continuous maps of surfaces into hyperbolic 3-manifolds with boundary. In Section 2, we review He's construction and introduce more general constructions which are utilized subsequently. In Section 3 , we introduce particularly interesting examples of singular surfaces to demonstrate the utility of the above techniques. In particular, we generate hyperbolic knots with arbitrarily small cusp density. In Section 4, we obtain various bounds for the category of alternating knots. In Section 5 we use an immersed twice-punctured Möbius band to prove that every closed orientable 3-manifold contains a knot whose complement is hyperbolic with maximal cusp volume at most 9. Furthermore, this knot can be made to have arbitrarily small cusp density.

Much of the research work for this paper was completed in the Williams SMALL program during the summer of 2001, with support from NSF Grants DMS-9820570, DMS-9803362, and Williams College and in the subsequent undergraduate thesis of the last-named author.

\section{MAPS OF SURFACES}

Here we introduce the terminology used in Ago00. Let $S$ be a surface of finite type ( $S$ may have both boundary and punctures), and let $f: S \rightarrow M$ be a continuous map such that every puncture maps properly into a cusp. This map might not necessarily be an embedding or an immersion. We will say that $f: S \rightarrow M$ is incompressible if every simple closed curve $c$ in $S$ for which $f(c)$ is homotopically trivial in $M$ bounds a disk in $S$. Let $U=[0, \infty) \times \mathbb{R}$. A boundary compression of $f$ is a proper map $b: U \rightarrow M$ such that there is a map $b^{\prime}: \partial U \rightarrow S$ with $f \circ b^{\prime}=b_{\mid \partial U}$, and $b^{\prime}(\partial U)$ is a proper simple line in $S$ which does not bound a properly embedded half-plane in $S$. The map $f$ is boundary incompressible if it has no boundary compression. The map $f: S \rightarrow M$ is essential if it is incompressible and boundary incompressible.

In [Thu79], Thurston introduced pleated surfaces. A map $f: S \rightarrow M$ is pleated if the boundary components of $S$ map to geodesics in $M$, and int(S) (the interior of $S$ ) is piecewise made of triangles which map under $f$ to subsets of $M$ that lift to 
ideal hyperbolic geodesic triangles in $\mathbb{H}^{3}$, so that the 1-skeleton forms a lamination in $S$. A pleated surface has an induced hyperbolic metric, where the lamination is geodesic.

In Ago00, Agol proved the following lemma.

Lemma 2.1. If $S$ is a pleated surface that is boundary incompressible, the sum of the lengths of the intersection curves in $S \cap \partial C$ is bounded above by $6|\chi(S)|$.

The idea is that for a pleated surface $S$ that is boundary incompressible, the intersection of the pleated surface with the cusps consists only of disjoint neighborhoods of the punctures. For otherwise, we could find a properly embedded arc in $S$ that was entirely contained in the pre-image in $S$ of the cusp. But then that arc could be homotoped through the cusp to the boundary of $M$. Hence, there would exist a boundary-compression.

Then the area in $S \cap C$ must be no more than $\frac{6}{2 \pi}$ of the total area of the surface, since this is the maximal density of a horocycle packing in $\mathbb{H}^{2}$. But the total area of $S$ is given by $2 \pi|\chi(S)|$. The area in $S \cap C$ is equal to the length of its boundary, yielding the lemma.

The fact that the sum of the lengths of these intersection curves is bounded above gives upper bounds on particular curves on $C$ as well as $V_{c}(M)$, as shown in Theorem 3.1 in Section 3

Let $K$ be a knot in $\mathbb{S}^{3}$ with hyperbolic complement. Let $C$ be a maximal cusp corresponding to the knot. If $K$ has an $n$ crossing projection $\pi(K)$, Z.-X. He obtains an $n$-punctured disk by connecting a single point placed below the plane of the projection to each point on the knot (see [He98). The result is an $n+1$ punctured sphere with $n$ meridinal punctures and a single (knotted, parallel to $K$ ) $\ell$-curve puncture. We will refer to such a surface as $S=H(\pi(K))$.

The surfaces resulting from He's construction may or may not be boundary incompressible. But He shows that his results would only be improved if any compressions or boundary-compressions are performed on $S$.

We now describe two methods we utilize to obtain interesting surfaces in knot complements. The first involves a mixture of He's construction and the Seifert algorithm for obtaining an orientable spanning surface.

Choose an oriented projection of a given knot $K$. Divide the projection sphere into finitely many different disk regions, intersecting only in their boundaries (the boundaries of which are transverse to the knot). Within each region, construct a link by connecting the endpoints of the knot leaving the region by arcs running parallel to the boundary of the region in such a way as to inherit an orientation on the link from the knot. (Two or more strands running along the boundary may be parallel to one another.)

Now perform either He's or Seifert's algorithm on the link in each region. Connect the resulting surfaces by bands across the region boundaries in such a way as to obtain a single contiguous surface with boundary equal to the original link.

We shall refer to surfaces constructed in this manner as Seifert-He surfaces. Seifert-He surfaces are not always incompressible or boundary incompressible, e.g. adding a nugatory crossing to any link and performing He's algorithm on the region containing this crossing will result in a boundary-compressible surface. Also, Seifert's algorithm does not always yield an incompressible surface when applied to nonalternating link projections. Furthermore, when we are using Seifert's algorithm on some parts of the knot and He's in other parts, we cannot be assured that 


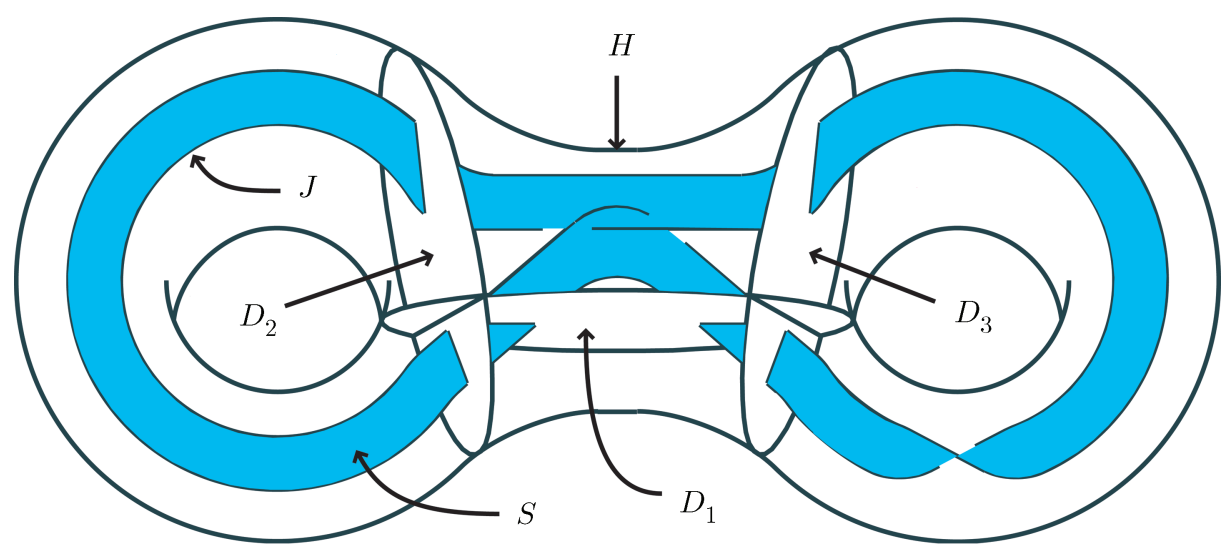

Figure 1. A knot $J$ in a handlebody bounding an immersed Twice-punctured Möbius band.

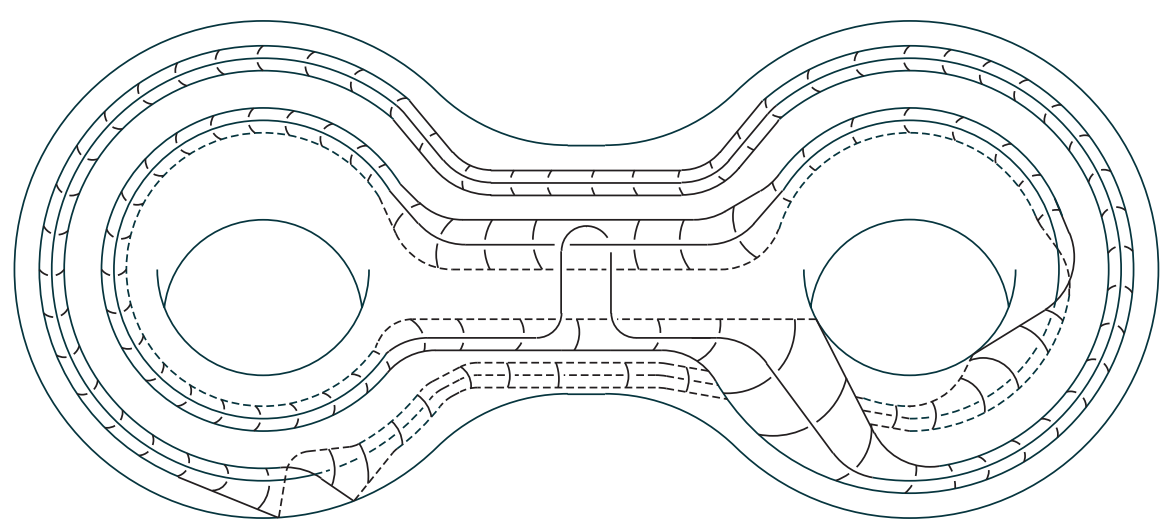

FiguRE 2. An essential annulus in $H-J$.

compressing our surface will improve our results as He can (since our surfaces may be of genus $g \geq 1$ rather than disks). We seek to address some of these difficulties subsequently.

A second technique we utilize involves placing the knot $K$ and surface $S$ inside a genus 2 handlebody $H$ and then showing that $S$ is incompressible and boundary incompressible in $H-K$. Then we embed $H$ appropriately in $\mathbb{S}^{3}$.

Let $J$ be the knot depicted in the handlebody $H$ appearing in Figure 1

Lemma 2.2. $H-J$ is hyperbolic and the immersed twice-punctured Möbius band $S$ is incompressible and boundary incompressible in $H-J$. The only essential annuli in $H-J$ have both boundary components on $\partial H$ and they avoid the disk $D_{1}$.

Proof. This knot in a handlebody was considered in AR93, where it was proved that $H-J$ is hyperbolic. There it was also shown that the only essential annuli in $H-J$ have both boundary components on $\partial H$, and that any essential annulus in $H-J$ avoids the disk $D_{1}$. An example from that paper of an essential annulus that does exist appears in Figure 2 , 


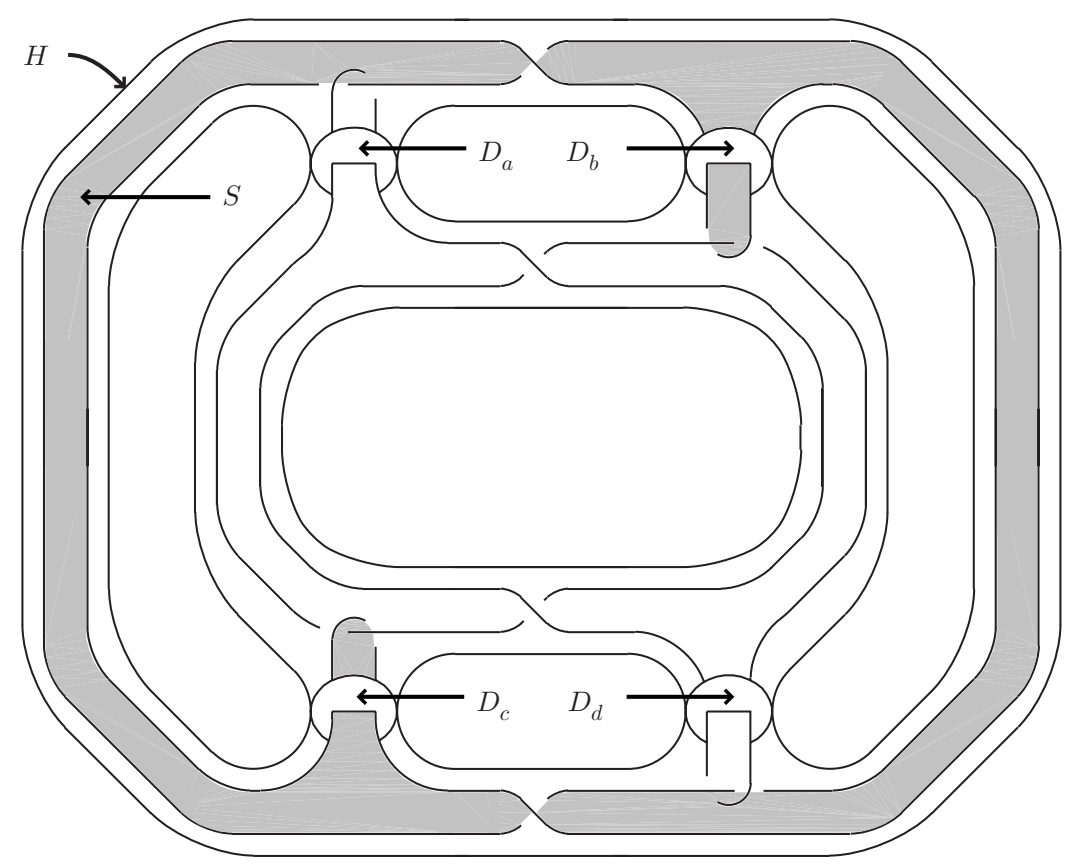

Figure 3. Creating a link that misses $D_{a}$.

We will show that the immersed twice-punctured Möbius band $S$ appearing in Figure 1 is essential in $H-J$. Suppose not. Lift $H$ to the genus five handlebody $H^{\prime}$ appearing in Figure 3. The knot $J$ lifts to the four component link $L$, and the Möbius band $S$ lifts to two embedded quadruply punctured annuli $A_{1}$ and $A_{2}$. A compressing or $\partial$-compressing disk for $S$ lifts to a compressing or $\partial$-compressing disk for either one of the two annuli, say $A_{1}$. By Dehn's Lemma, there must be an embedded disk intersecting $A_{1}$ only in its boundary, or intersecting $A_{1}$ and the boundary of a neighborhood of the link only in its boundary. Suppose $E$ were such a disk. We prove that $E$ can be chosen so as not to intersect any of $D_{a}, D_{b}, D_{c}$ or $D_{d}$. Suppose that $E$ did intersect one of them, say $D_{a}$ for convenience. If $E$ intersects $D_{a}$ in a trivial curve on $D_{a}$, then we can isotope to remove the intersection. If $E$ intersects $D_{a}$ in a curve that wraps once around one of the punctures, then there would be a disk in the link complement bounded by a meridian of one of the link components, which cannot occur. If $E$ intersects $D_{a}$ in a simple closed curve that wraps around both punctures on $D_{a}$, then we can cut the link component open at its intersection with $D_{a}$ and cap off each component with a straight line segment on $D_{a}$, as in Figure 3 ,

The resulting link complement does not intersect $D_{a}$, so taking a disk on $D_{a}$ with a disk on $E$ yields a sphere that separates one of these link components from the rest of the link. But as a link in $\mathbb{S}^{3}$, this link is nonsplittable. Hence there are no simple closed intersections of $E$ with $D_{a}$, or for that matter with $D_{b}, D_{c}$ or $D_{d}$. Already, we have eliminated all possible intersections of $E$ with $D_{c}$ and $D_{d}$. We now consider arcs of intersection of $E$ with $D_{a}$. Let $\mu$ be the arc of intersection between $A_{1}$ and $D_{a}$, and let $D^{\prime}$ be the once-punctured disk that $\mu$ cuts off $A_{1}$. Let $A^{\prime}$ be the annulus in $N(L)$ that is cut off from $N(L)$ by $D_{a}$ and that intersects $D^{\prime}$. Each 
arc of intersection between $D_{a}$ and $E$ must then begin and end on $\mu \cup\left(A \cap D_{a}\right)$. Let $x$ be such an arc cutting a disk $E^{\prime}$ off $E$. Let $g$ be the arc of intersection of $E^{\prime}$ with $D^{\prime} \cup A$. If $g$ does not loop around the puncture on $D^{\prime}$, we can slide $g$ down $D^{\prime}$ and push it through $\mu$, eliminating this intersection of $E$ and $D^{\prime}$. If $g$ does loop around the puncture, then the union of $E^{\prime}$, a subdisk of $D^{\prime} \cup A^{\prime}$ cut off by $g$ and a subdisk of $D_{a}$ cut off by $\mu$ and $x$, yields a once-punctured sphere, a contradiction. Hence there are no compressing or $\partial$-compressing disks for $A_{1}$ that intersect $D_{a}$, or for that matter any of the four disks. There can also be no such disks that have boundary in $D^{\prime} \cup A^{\prime}$ or the three other disks with annuli cut off by $D_{b}, D_{c}$ and $D_{d}$, as any such would have boundary homotopic to a meridian of the link, which is not possible. Let $L^{\prime}$ be the sublink of $L$ that forms the boundary of $A_{1}$. Let $D^{\prime \prime}$ be the punctured disk cut off of $A_{1}$ by $D_{b}$, and let $A^{\prime \prime}$ be its corresponding annulus in $N\left(L^{\prime}\right)$. The only possibility remaining is for $E$ to lie in $A_{1} \cup N\left(L^{\prime}\right)-\left(D^{\prime} \cup A^{\prime} \cup D^{\prime \prime} \cup A^{\prime \prime}\right)$. But $E$ must lie in $H^{\prime}-\left(L \cup N\left(D_{a} \cup D_{b} \cup D_{c} \cup D_{d}\right)\right)$. This is a solid torus with eight trivial arcs removed, so it is a genus nine handlebody. Each of the generators of the fundamental group of $A_{1} \cup N\left(L^{\prime}\right)$ that would yield a loop that does not intersect $D_{a} \cup D_{b} \cup D_{c} \cup D_{d}$ are nontrivial generators for this handlebody. Since the fundamental group of a handlebody is a free group on its generators, any word in these generators would yield a nontrivial element of $\pi_{1}\left(H^{\prime}-\left(L \cup N\left(D_{a} \cup D_{b} \cup D_{c} \cup D_{d}\right)\right)\right.$. Since no compression or $\partial$-compression disks intersect $D_{a} \cup D_{b} \cup D_{c} \cup D_{d}$, this word is also nontrivial in $H^{\prime}-L$. This completes the proof that $A_{1}$ is essential in $H^{\prime}-L$ and therefore that $S$ is essential in $H-J$.

\section{EXAMPLES OF SINGULAR SURFACES}

Many examples of singular surfaces occur in knot complements. In the following examples, such surfaces will be used to find bounds on cusp volume, meridian length, and $\ell$-curve length.

Example 1. We can utilize the He surface (see Section 2) $S=H(\pi(K)$ ) for a projection $\pi(K)$ of a hyperbolic knot to find bounds.

Theorem 3.1. Let $K$ be a knot with c crossings in projection $\pi(K)$, and let $|m|,|\ell|$ denote the lengths of the meridian $m$ of the knot and the shortest $\ell$-curve on the maximal cusp. The following bounds hold for $K$ and its maximal cusp $C$ :

i) $|m| \leq 6-\frac{7}{c}$

ii) $|\ell| \leq 5 c-6$,

iii) $V_{C}(M) \leq \frac{9 c}{2}\left(1-\frac{1}{c}\right)^{2}$.

If $S$ is boundary incompressible, the argument is relatively straightforward and appears in the proof below. If $S$ is not boundary incompressible, performing the corresponding boundary compression will only improve the bounds in Theorem 3.1 . However, we need the following lemma to prove this.

Lemma 3.2. Let $S$ be the proper continuous image of a punctured disk with $c$ meridinal boundary components and exactly one $\ell$-curve boundary component such that $S$ may be boundary-compressed along an arc $\alpha \subset S$. Then performing this boundary compression will result in a surface $S^{\prime}$ such that the genus of $S^{\prime}$ remains $0, S^{\prime}$ has strictly fewer boundary components than $S$, and $S^{\prime}$ has exactly one $\ell$-curve boundary component. 


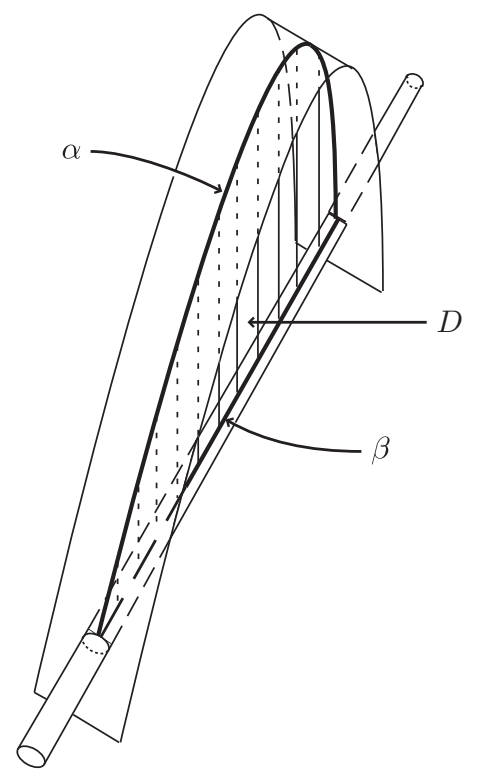

FiguRE 4 . The boundary-compressing disk $D$ with boundary $\partial D=\alpha \cup \beta$ is shown here.

Proof. Since $S$ is the image of a surface with $c+1$ punctures such that exactly one of the punctures corresponds to an $\ell$ curve on the cusp and $c$ of the punctures correspond to meridinal curves on the cusp boundary, each possible $\partial$-compressing arc $\alpha$ naturally falls into one of four categories:

(1) $\alpha$ connects a meridian to itself,

(2) $\alpha$ connects one meridian $m_{1}$ to another $m_{2}$,

(3) $\alpha$ connects the $\ell$-curve boundary to a meridian,

(4) $\alpha$ connects the $\ell$-curve boundary to itself.

We will discuss only the second possibility in detail since the arguments for each are similar. See Kat02 for complete details. In this case, form an annulus $A$ by connecting the ends of $N(\alpha)$ to each other by a disk in $C$ which does not intersect $l, m_{1}$, or $m_{2}$ in its interior. Define the number of half-twists in $N(\alpha)$ to be the number of half-twists in $A$ as an annulus embedded in $S^{3}$. See Figure 4 for a picture of the case that $N(\alpha)$ has zero half-twists.

Note that if $N(\alpha)$ has an even number of half-twists, then performing a boundarycompression along it will result in a single punctured disk $S^{\prime}$ which has one $\ell$ curve boundary component and one fewer boundary component. In particular, one boundary component will be trivial, which we can cap off with a disk. The resulting surface has two fewer meridianal boundary components. But note that the result has at least one meridianal puncture remaining, as otherwise $\partial D$ would homotope to $\ell \subset \partial S$, which is a contradiction since a nontrivial knot cannot bound a disk.

In the case that $N(\alpha)$ has an odd number of half-twists, the boundary-compressing disk $D$ will intersect $S$ but we can still perform the boundary-compression, as in Figure 5. The resulting surface will have one fewer boundary component than $S$ and it will have one boundary component that wraps twice around a meridian. 


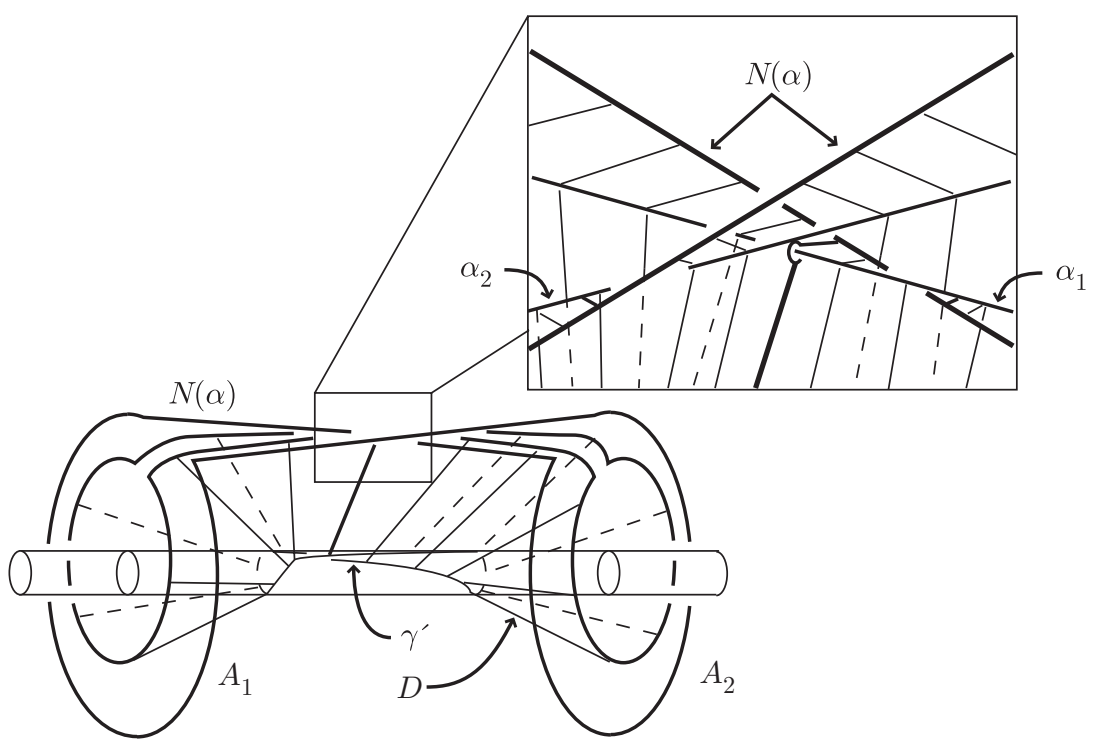

Figure 5. Performing a boundary compression on a surface whose constructed band $B=N(\alpha) \cup A_{1} \cup A_{2}$ has a single half-twist. Notice that $\gamma^{\prime}$, the boundary component which replaces $\gamma_{1}$ and $\gamma_{2}$, is the product $\gamma_{1} \circ \gamma_{2}$.

Proof of Theorem 3.1. First, we construct a He surface $S$ in $\mathbb{S}^{3}-K$ and notice that $S$ has $c+1$ boundary components, $c$ corresponding to meridinal punctures and one corresponding to an $\ell$-curve. By Lemma 3.2, we know that there is a boundary incompressible punctured disk $S$ with one $\ell$-curve boundary and no more meridianal boundary than the initial surface. (We may have turned two different meridianal boundary components into a single double meridian boundary component for instance.) Taking a pleated representative of this surface, we know by Lemma 2.1 that the sum of the areas of the regions of intersection between $S$ and $N(K)$ will be less than or equal to $6|\chi(S)|$. Hence,

$$
c|m|+|\ell| \leq 6|c-1| .
$$

From this, we see

$$
|m| \leq 6-\frac{6+|\ell|}{c} .
$$

Since $|\ell| \geq 1$, we know that

$$
|m| \leq 6-\frac{7}{c}
$$

We also see

$$
|\ell| \leq(6-|m|) c-6 .
$$

Since $|m| \geq 1$, we have

$$
|\ell| \leq 5 c-6,
$$

and then we have that

$$
V_{C}(M) \leq \frac{|m||\ell|}{2} \leq \frac{1}{2}|m|((6-|m|) c-6) .
$$



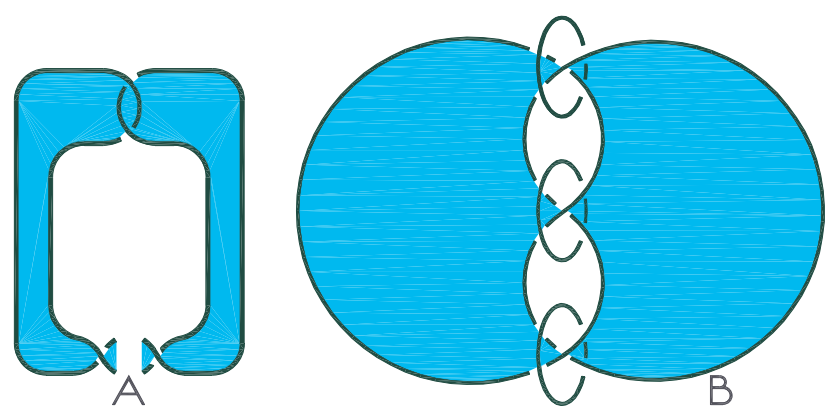

Figure 6. Two immersed surfaces.

Maximizing this with respect to $|m|$, we find that

$$
V_{C}(M) \leq \frac{9 c}{2}\left(1-\frac{1}{c}\right)^{2}
$$

Example 2. Let $K$ be a twist knot, and consider the surface obtained by coning a clasp of $K$ to a point below the plane of the knot and then affixing a band to the resulting pair of punctured disks as in Figure [6. The resulting twice-punctured disk intersects the cusp meridian twice and $\ell$ once. It is boundary incompressible as if it did boundary-compress; we would have an essential annulus in the knot complement, which cannot occur for a hyperbolic 3-manifold. The surface has Euler characteristic $\chi=-1$ yielding

$$
2|m|+|\ell| \leq 6|-1|
$$

Maximizing $|m||\ell|$ subject to the above restraint yields $|m||\ell| \leq 9 / 2$ which gives an upper bound of $9 / 4$ on cusp volume. Note that if we knew the angle between an $|m|$ curve and an $|\ell|$ curve on the horosphere centered at $\infty$ in the universal cover of $\mathbb{S}^{3}-K$, then we could produce better bounds on the maximal cusp volume. It is known that the cusp volumes of the twist knots approach 2 from below, but we include this example as an illustration.

Example 3. By considering the embedded surface shown for the link in Figure $6 \mathrm{~B}$ we obtain $|\ell| \leq 6$. The surface does not intersect the meridian of the cusp, so no bounds on meridian length or volume are obtained. It is incompressible and boundary incompressible since it is a Seifert surface of minimal genus for the trefoil knot. Measurements from SNAPPEA Wee show that this upper bound is realized, and $|\ell|=6$ in this case. More generally, a genus $g$ knot must have the length of some $\ell$-curve bounded above by $12 g-6$.

Example 4. Consider the twice-punctured Möbius band $S$ bounded by the knot $K$ in the genus 2 handlebody $H$ shown in Figure 1, Let $T$ be the homeomorphism given by a full twist of the disk $D_{1}$ (isotoping so that the only the area of $H$ changed by $T$ is the area $A$ containing the punctures of $S$ and bounded by the disks $D_{1}, D_{2}$, and $D_{3}$ ). Define another homeomorphism $R$ similarly by performing a full twist of $D_{2}$ followed by isotopy to push all changed areas into region $A$ and then doing a full twist of $D_{3}$ and isotoping all changes to area $A$ again. Since 


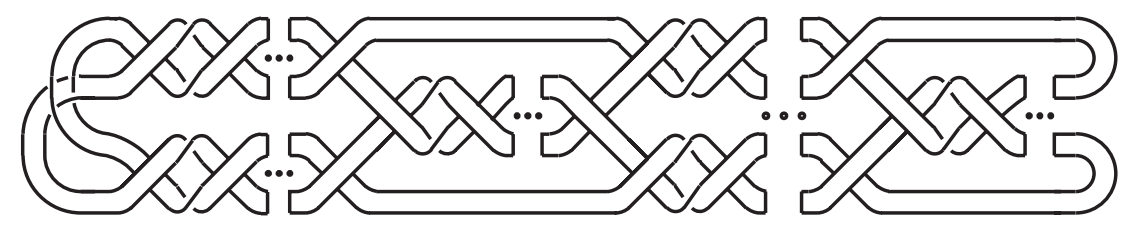

FIGURE 7. Knot obtained by twisting disks in handlebody.

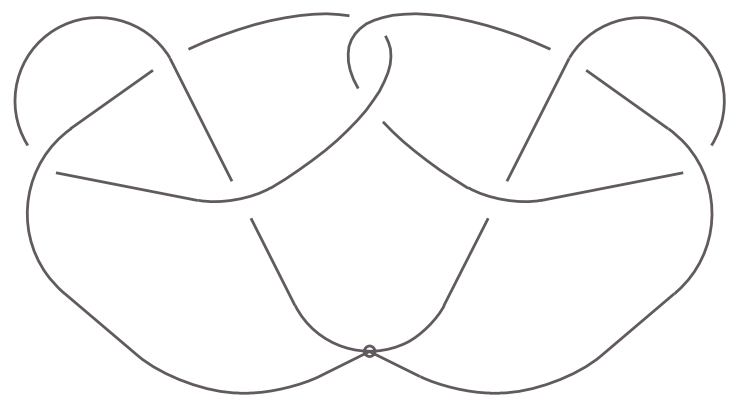

FiGURE 8. Graph representing a special handlebody.

$R$ and $T$ are homeomorphisms, they preserve the incompressibility and boundary incompressibility of the Möbius band $S$ inside $H$ that we showed in Lemma 2.2. By applying a transformation of the form $T^{n}\left(R^{n} T^{n}\right)^{p}$, the knot $K$ can be made to resemble the one appearing in Figure 7 . When $n$ is large, the volume of this knot approaches the volume of the link obtained by surrounding each of the individually twisted parts by an additional individually trivial link component. When $p$ is large, this link can be made to have an arbitrarily large number of cusps so that the resulting knot complement can be made to have arbitrarily high volume.

By embedding $H$ in a carefully chosen handlebody (for example, the one obtained from thickening the graph in Figure 8) in $\mathbb{S}^{3}$ we are assured that $S$ is incompressible and boundary incompressible in $\mathbb{S}^{3}-K$ (even after performing $R$ and $T$ as many times as desired). Since $R$ and $T$ only change the way $S$ is embedded in space but do not affect Euler characteristic, we can obtain a bound on cusp volume.

The immersed surface formed by the twice-punctured Möbius band has Euler characteristic $\chi=-2$ and intersects the meridian of the cusp twice and $l$ once to give

$$
2|m|+|\ell| \leq 6|-2|
$$

resulting in an upper bound on cusp volume, $V_{c} \leq 9$. As explained above, the knot $K$ can be made to have arbitrarily large volume while preserving the Möbius band $S$, so that $K$ can be made to have arbitrarily low cusp density.

\section{Alternating Knots}

In this section we provide bounds that apply to alternating knots.

Theorem 4.1. Let $K$ be an alternating knot in $\mathbb{S}^{3}$ with $c$ crossings. Then:

(1) $|m| \leq 3-6 / c$,

(2) $|l| \leq 3 c-6$,

(3) $V_{c}(M) \leq \frac{9 c}{2}(1-2 / c)^{2}$. 
The cusp volume bound can be compared with results of Lackenby Lac01]. The twist number of an alternating knot projection is the number of maximal chains of end-to-end bigonal regions appearing in the diagram where a crossing that does not appear on a bigon counts as an individual twist. Lackenby (together with an improvement of the upper bound due to Ian Agol and Dylan Thurston) proved that if $t$ is the twist number of a reduced alternating diagram for a prime hyperbolic knot, then $\frac{v_{3}}{2}(t-2) \leq \operatorname{Vol}\left(\mathbb{S}^{3}-K\right) \leq 10 v_{3}(t-1)$, where $v_{3}$ is the Gieseking constant, approximately 1.01494. An immediate consequence of this is that $V_{c}(K) \leq \frac{\sqrt{3}}{2 v_{3}}\left(\mathrm{Vol}\left(\mathbb{S}^{3}-K\right) \leq 5 \sqrt{3}(t-1)\right.$. Note that as the ratio of crossings to twist number increases, the Lackenby result gives the better bound. However, for projections where $c \leq 2.26(t-1)$, Theorem 4.1 gives the lower bound.

We can use these results to examine systole length, which is the length of the shortest closed geodesic in a manifold.

In [AR02, it was proved that the complement of a hyperbolic knot in $\mathbb{S}^{3}$ has systole length less than 7.35534. The following corollary is an immediate consequence of the bound on $|m|$ obtained above and Theorem 3.2 from that paper.

Corollary 4.2. If $K$ is a hyperbolic alternating knot in $\mathbb{S}^{3}$, the systole length of $\mathbb{S}^{3}-K$ is less than 4.5 .

To prove Theorem 4.1, we will look at the so-called checkerboard surfaces corresponding to a given projection of a knot. These are two surfaces obtained by coloring regions of the projection plane alternately black and white. Since four regions meet at a crossing, two black regions and two white regions meet at each crossing. We connect the two like-colored regions by a twisted band passing through the crossing. The two surfaces will intersect in a vertical arc at each crossing. Note that the boundary of each surface is the knot and the surfaces can either be orientable or nonorientable. In MT93, the authors prove that in the case of prime alternating knots, the checkerboard surfaces corresponding to any reduced alternating projection are incompressible. As they state, this proof can be extended to prove boundary-incompressibility as well.

Proof of Theorem 4.1. Let $S_{1}$ and $S_{2}$ be the two checkerboard surfaces for $K$ in a particular reduced alternating projection. Note that the boundaries of $S_{1}$ and $S_{2}$ together form a diamond pattern on the boundary of the maximal cusp boundary, which we call a harlequin tiling. See Figure 9 .

The union of the the boundaries of $S_{1}$ and $S_{2}$ can be decomposed into $2 c$ meridians, two meridians coming from the boundary of each of the top diamonds, or it can be decomposed into two $\ell$-curves. Notice that we allow ourselves to jump from one surface to the other in breaking up and reconstructing these curves.

In determining the Euler characteristic of $S_{1}$ and $S_{2}$, we can treat the regions in the projection plane as the faces of a decomposition, inserting vertical edges at each crossing and vertices at the top and bottom of each of these edges. We then have a decomposition of the two surfaces with $4 c$ vertices, $6 c$ edges and $f$ faces, where $f=c+2$. Then since each has nonpositive Euler characteristic,

$$
\left|\chi\left(S_{1}\right)\right|+\left|\chi\left(S_{2}\right)\right|=|4 c-6 c+(c+2)|=c-2 .
$$




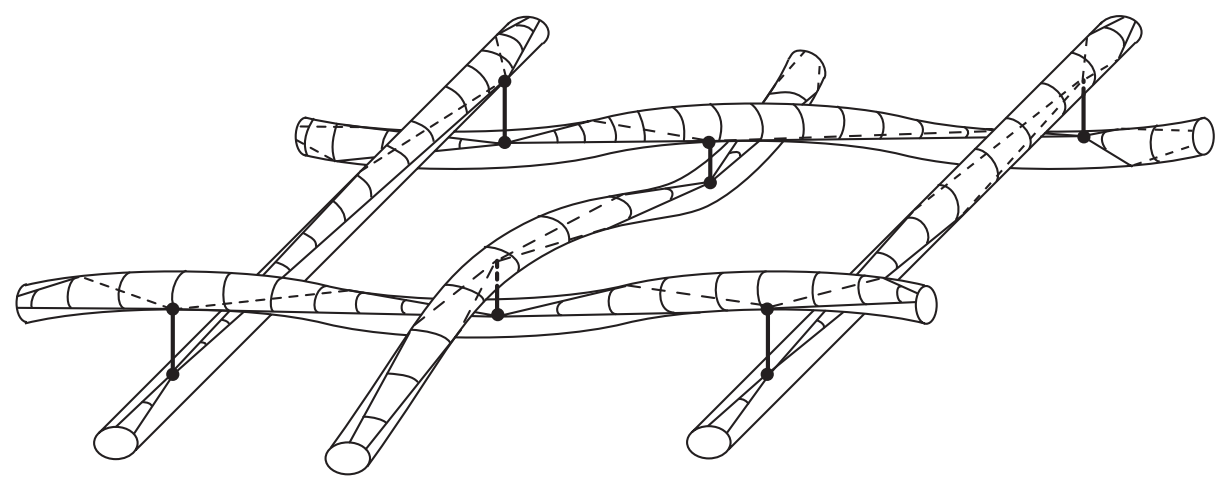

Figure 9. The induced harlequin tiling on $\partial C$.

Thus, by Lemma 2.1] we obtain

$$
\begin{gathered}
2 c|m| \leq 6\left(\left|\chi\left(S_{1}\right)\right|+\left|\chi\left(S_{2}\right)\right|\right) \leq 6(c-2), \\
|m| \leq 3-6 / c
\end{gathered}
$$

and

$$
\begin{aligned}
2|\ell| & \leq 6(c-2), \\
|\ell| & \leq 3 c-6 .
\end{aligned}
$$

By maximizing $f(|m|)=|m||\ell|$ as a function of $|m|$, we obtain

$$
V_{c}(M) \leq \frac{9 c}{2}(1-2 / c)^{2} .
$$

\section{An APPLICATiON}

In this section, we use immersed surfaces to prove the following:

Theorem 5.1. Any closed orientable 3-manifold $M$ contains a knot $K$ such that $M-K$ is hyperbolic with cusp volume no larger than 9 .

Proof. Let $M$ be a closed orientable 3-manifold. In Mye82 (see also [Ada94 for an alternative proof), Myers proved that every such manifold contains a knot such that its complement in $M$ is hyperbolic. Let $J^{\prime}$ be such a knot in $M$. Define a vertical geodesic in $M$ to be a geodesic that is perpendicular to the cusp boundary at each of its ends. Note that there are infinitely many distinct vertical geodesics in any cusped finite volume hyperbolic 3-manifold. Although a vertical geodesic need not be embedded in the manifold, if it is not, it will intersect itself at most a finite number of times. At those points of intersection, we can perturb the geodesic slightly to remove the self-intersections. We will do the perturbation to avoid introducing any local tangles. Given a particular vertical geodesic $\alpha$, shrink the cusp back until it only intersects $\alpha$ at its ends. If $\alpha$ is an unknotting tunnel for $M-J^{\prime}$, replace it with a vertical geodesic that is not. By Joh95, there are only finitely many unknotting tunnels up to isotopy in a hyperbolic 3-manifold. Moreover, there is only one such in each homotopy class. So we can find a vertical geodesic $\alpha$, possibly perturbed, that is not an unknotting tunnel. 
Let $M^{\prime}=M-N\left(J^{\prime} \cup \alpha\right)$. Then $M^{\prime}$ has a genus two boundary. We will prove that $M^{\prime}$ is hyperbolic. That is to say, it is irreducible, $\partial$-irreducible and contains no essential tori. Note that we are not claiming there are no essential annuli.

To see irreducibility, suppose $S$ is an essential sphere in $M^{\prime}$. Since $M^{\prime} \subset M-J^{\prime}$, and $M-J^{\prime}$ is hyperbolic, $S$ bounds a ball in $M-J^{\prime}$. Therefore, it bounds a ball in $M^{\prime}$ as well.

To see boundary irreducibility, suppose $\partial M^{\prime}$ is compressed. One or two tori result from the compression. Let $T$ be one of them. Since $T$ sits in $M-J^{\prime}$, which contains no essential tori, it must either be boundary parallel in $M-N\left(J^{\prime}\right)$, or it must compress. If it is boundary-parallel, then $\alpha$ can be homotoped into $\partial\left(M-N\left(J^{\prime}\right)\right)$. Hence $\alpha$ would lift to an arc that connects one horoball to itself, rather than one horoball to another, as we know $\alpha$ does. So $T$ must compress. In the case that the original compression yielded two tori, we can replace that compression with this one, so we need only concern ourselves with a single torus.

As above, it is still the case that this torus $T$ compresses. Since the sphere $S$ resulting from the compression of $T$ must bound a ball $B$ in $M-J^{\prime}$, we know that $T$ bounds either a knot exterior or a solid torus in $M-J^{\prime}$.

In the first case, let $D$ be the compressing disk and let $R$ be the knot exterior. Then $R \cup D=B$, and $\alpha$ must puncture $D$ at least once, since $T$ is incompressible in $M^{\prime}$. The ball $B$ lifts from $M-J$ to a disjoint set of balls in $\mathbb{H}^{3}$. Let $B^{\prime}$ be one such ball. Then $B^{\prime}$ must contain a homeomorphic copy of $R$, call it $R^{\prime}$. The boundary of $R^{\prime}$ must be incompressible in $\mathbb{H}^{3}-p^{-1} N\left(J^{\prime} \cup \alpha\right)$. Since $B$ and therefore $B^{\prime}$ has finite volume, we can choose a 3-ball $E \in \mathbb{H}^{3}-p^{-1} N\left(J^{\prime}\right)$ that contains $B^{\prime}$ and such that any arc in $p^{-1}(\alpha)$ that is intersected by $E$ is isotopic into $\partial E \in E$. Then the fundamental group of $E-p^{-1}(N(\alpha))$ is a free group on a finite number of generators. But $R^{\prime}$ is a knot exterior contained within it with incompressible boundary. But then the fundamental group of $R^{\prime}$ is a subgroup of a free group, meaning it is free itself by the Nielsen-Schreier Subgroup Theorem. However, nontrivial knot exterior groups are not free, a contradiction.

Hence $T$ bounds a solid torus in $M-J^{\prime}$ and therefore in $M^{\prime}$. This implies that $M^{\prime}$ is a handlebody, contradicting our assumption that $\alpha$ was not an unknotting tunnel. So $\partial M^{\prime}$ is incompressible.

To see that there are no essential tori in $M^{\prime}$, suppose that $T$ is such a torus. As above, $T$ must bound a solid torus in $M-J^{\prime}$. However, then it does so in $M^{\prime}$ as well, a contradiction.

Let $H$ be the handlebody that appears in Figure 1, and $J$ the knot within it. By Lemma 2.2, $H-J$ is hyperbolic with an immersed incompressible boundary incompressible twice-punctured Möbius band contained within it. Also, any essential annuli in $H-J$ have both boundary components on $\partial H$ and they do not intersect the disk $D_{1}$.

We will glue the boundary of the handlebody $H$ to the boundary of $M^{\prime}$ in order to obtain $M$ again. Note that we can include twists in the handles when we do so, without changing the topological type of $M$. The result will yield an embedding of the knot $J$ in $M$. Call that embedded knot $K$. Let $Q$ be the genus two surface in $M$ corresponding to $\partial H$.

The immersed Möbius band $S$ is essential in $M-K$ since $S$ is essential in $H-L$ and $\partial M^{\prime}$ is incompressible in $M^{\prime}$. 
We now show that $M-K$ is hyperbolic. Irreducibility follows from irreducibility of $M^{\prime}, H-J$ and incompressibility of $S$ to either side. Boundary-irreducibility follows from the incompressibility of $\partial N(J) \in H$ and the incompressibility of $\partial M^{\prime} \in$ $M^{\prime}$.

We must show that $M-K$ is atoroidal. Since $H-J$ and $M^{\prime}$ are each atoroidal, any essential torus $T$ in $M-K$ must intersect $S$. By minimizing the intersections, we can assume that $T$ intersects $M^{\prime}$ and $H-J$ in essential annuli, each of the boundary components of which lie on $Q$. But the boundary components of any essential annulus in $H-J$ do not intersect $D_{1}$. In particular, in order to construct an essential torus with such an annulus and an annulus in $M^{\prime}$, it would be necessary for the annulus in $M^{\prime}$ to be isotopic to an annulus that avoids $\partial N(\alpha)$. However, such an annulus in $M^{\prime}$ then exists as an annulus in $M-N\left(J^{\prime}\right)$. Since $M-N\left(J^{\prime}\right)$ is hyperbolic, the annulus must be boundary-parallel in $M-N\left(J^{\prime}\right)$. But then $\alpha$ can be homotoped to the boundary, contradicting our choice of $\alpha$ as an arc that connects two distinct horoballs. Hence no essential tori can be constructed. Thus, $M-K$ is hyperbolic with an immersed incompressible boundary incompressible twice-punctured Möbius band. Therefore, by results in Example 4 from Section 2, we see that the maximal cusp volume must be at most 9 .

Corollary 5.2. Any closed orientable 3 -manifold $M$ contains a knot $K$ such that $M-K$ is hyperbolic with arbitrarily low cusp density and cusp volume at most 9.

Proof. Using exactly the same argument, but twisting up the knot inside the handlebody as in Example 4, before the handlebody is inserted into the manifold, yields a hyperbolic knot in the manifold with complement of arbitrarily high volume but cusp volume bounded by 9 .

In particular, any counterexample to the Poincaré Conjecture must come from Dehn filling a one-cusped hyperbolic 3-manifold with cusp volume at most 9 (with arbitrarily low cusp density).

\section{REFERENCES}

[Ada94] Colin Adams, Dehn filling hyperbolic 3-manifolds, Pacific J. Math. 165 (1994), no. 2, 217-238. MR1300832 (95h:57018)

[Ada02] W Waist size for cusps in hyperbolic 3-manifolds, Topology 41 (2002), no. 2, 257270. MR1876890 (2003e:57023)

[Ago00] Ian Agol, Bounds on exceptional Dehn filling, Geom. Topol. 4 (2000), 431-449. MR.1799796 (2001j:57019)

[AR93] Colin Adams and Alan Reid, Quasi-fuchsian surfaces in hyperbolic knot complements, J. Austral. Math. Soc. (Series A) 55 (1993), 116-131. MR1231698 (95a:57004)

[AR02] - Systole length in hyperbolic 3-manifolds, Math. Proc. Cambridge Philos. Soc. 128 (2002), no. 1, 103-110. MR.1724432 (2000h:53049)

[He98] Zheng-Xu He, On the crossing number of high degree satellites of hyperbolic knots, Math. Res. Lett. 5 (1998), no. 1-2, 235-245. MR1617901 (99c:57023)

[Joh95] Kurt Johannson, Topology and combinatorics of 3-manifolds, Springer-Verlag, 1995. MR.1439249 (98c:57014)

[Kat02] Eric Katerman, Singular maps of surfaces into hyperbolic 3-manifolds, Undergraduate Thesis (2002), 1-63.

[Lac00] Marc Lackenby, Word hyperbolic Dehn surgery, Invent. Math. 140 (2000), 243-282. MR:1756996 (2001m:57003)

[Lac01] , The volume of hyperbolic alternating knot complements, Proc. London Math. Soc. (3) 88 (2004), no. 1, 204-224. MR2018964 (2004i:57008) 
[MT93] William Menasco and Morwen Thistlethwaite, The classification of alternating links, Ann. of Math. 138 (1993), no. 1, 113-171. MR1230928 (95g:57015)

[Mye82] Richard Myers, Simple knots in compact orientable 3-manifolds, Trans. of A.M.S. 273 (1982), 75-91. MR0664030 (83h:57018)

[Thu79] William Thurston, The geometry and topology of three-manifolds, Princeton University Press, 1979.

[Wee] Jeffery Weeks, Snappea, A computer program for creating and studying hyperbolic 3manifolds, available at http://www.geometrygames.org.

Department of Mathematics, Williams College, Williamstown, Massachusetts 01267

E-mail address: Colin.Adams@williams.edu

Francis W. Parker School, Chicago, Illinois 60614

E-mail address: acolestock@fwparker.org

Department of Mathematics, University of Chicago, Chicago, Illinois 60637-1538

E-mail address: fowler@math.uchicago.edu

Department of Mathematics, Columbia University, New York, New York 10027

E-mail address: wgillam@math.columbia.edu

Department of Mathematics, University of Texas, Austin, Texas 78712

E-mail address: katerman@mail.utexas.edu 